\title{
Distal Chevron Osteotomy vs The Simple, Effective, Rapid, Inexpensive Technique (SERI) for Mild to Moderate Isolated Hallux Valgus: A Randomized Controlled Study
}

\section{Ezequiel Palmanovich ( $\nabla$ ezepalm@gmail.com )}

Meir Medical Center - Associated to Sackler Faculty of Medicine. Tel Aviv University. Israel https://orcid.org/0000-0002-6068-9554

Nissiom Ohana* - equal first author contribution

Meir Medical Center

llan Small

Tel Aviv Sourasky Medical Center

Iftach Hetsroni

Meir Medical Center

Eyal Amar

Tel Aviv Sourasky Medical Center

\section{Zachary T Sharfman}

Montefiore Medical Center

David Segal

Meir Medical Center

\section{Ran Atzmon}

Assuta Medical Center

Research article

Keywords: SERl; Chevron; Minimal Invasive Surgery; Hallux Valgus

Posted Date: March 13th, 2020

DOl: https://doi.org/10.21203/rs.3.rs-17170/v1

License: (9) (1) This work is licensed under a Creative Commons Attribution 4.0 International License.

Read Full License 
Version of Record: A version of this preprint was published at Indian Journal of Orthopaedics on August 8th, 2020. See the published version at https://doi.org/10.1007/s43465-020-00209-0. 


\section{Abstract}

Background Hallux valgus is a common foot deformity that leads to functional disability with serious sequelae. Minimally invasive surgery is often used to treat hallux valgus in order to reduce wound complications and improve recovery time. The objective of this study was to compare a Simple, Effective, Rapid, Inexpensive (SERI) technique with a simple Chevron technique in patients with minimum of oneyear follow. Methods and Materials Between the years 2014-2015 we performed a prospective study comparing the SERI minimally invasive technique to treat symptomatic hallux valgus with a standard chevron osteotomy technique. All procedures were performed by a single fellowship trained foot and ankle surgeon. Twenty-one patients were randomized to the SERI cohort and 15 to the standard Chevron technique. Results The mean pre-operative intermetatarsal angle (IMA) of the SERI group was $14.8 \pm 1.9$ (11.9 - 22.9). The mean pre-operative IMA of the Chevron control group was $13.3 \pm 2.3(10.4-18.2)(p=$ 0.038). The mean IMA two weeks after surgery was $6.0 \pm 2.3$ (2.4-12) in the SERI group, and $6.1 \pm 3$ (2.613.1) in the control group. At the two week and one year follow up, there was no significant difference found in the IMA between the two groups $(p=0.871)$. Neither groups reported symptomatic transfer metatarsalgia throughout the follow up period. The SERI group had increased metatarsophalangeal joint (MTPJ) motion $(p<0.001)$ however, all other parameters with similar. Conclusion The SERI technique provided comparable outcomes at up to one year follow up when compared to a standard Chevron osteotomy for moderate hallux valgus. This study demonstrated good reproducible results using the SERI technique for moderate hallux valgus.

\section{Introduction}

Hallux Valgus is an acquired anatomical deformity of the foot. The pathological process starts with a medial deviation of the first metatarsal at the first metatarsophalangeal joint (MPJ), lateral deviation of the first phalanx, and subluxation of the metatarsophalangeal joint ${ }^{1}$. Patients with symptomatic hallux valgus that fail conservative management are typically indicated for operative intervention based on weight bearing standard radiographic evaluation of hallux valgus. Weight bearing standard radiographic evaluation of hallux valgus is based on the intermetatarsal angle (IMA) hallux valgus angle (HVA), Distal Metatarsal Articular Angle (DDMA), and Hallux Valgus Interphalangeal angle (HVI) ${ }^{2}$. Hallux valgus is classified as mild when the IMA is between 9-12 degrees and the HVA $\leq 40$, moderate with an IMA between 13-15 degrees and HVA $>40$, and severe with an IMA above 20 degrees and HVA $>41^{2}$.

Risk factors for the development of hallux valgus include family history, connective tissue disorders, pes planus, age, hypermobility of the first-ray, and the use of high-heeled shoes ${ }^{3}$. Hallux valgus may cause impaired function of the first metatarsophalangeal joint with bursitis or callosities, pain, and transfer metatarsalgia ${ }^{1}$. Pain may develop due to irritation of the dorsal cutaneous nerve and inflammation of the bursa over the medial eminence ${ }^{3}$.

Various surgical procedures have been successfully used to treat mild to moderate hallux valgus including distal chevron osteotomies. The goals of surgery in these cases includes reorientation of the 
first MPJ and first ray ${ }^{1}$. The distal chevron osteotomy has been associated with complications such as pain, fibrosis, hallux valgus recurrence, transfer metatarsalgia, and osteonecrosis of the metatarsal head 1,3-6. Furthermore, whenever operating in the foot, wound complications are common and serious complications.

In an attempt to avoid these complications, we employed a SERI technique, which is similar to a percutaneous distal metatarsal osteotomy (PDO) described by Magnan et al ${ }^{7}$. While a PDO has a distal incision, the SERI technique uses a proximal incision. The advantages of the SERI technique include reduced recovery and rehabilitation times, reduced revisions surgeries, and avoiding lateral cortex penetration ${ }^{1-3,6}$. Furthermore, there is minimal soft tissue dissection, which decreases the risk of vascular damage as the technique causes minimal capsular disruption, ultimately resulting in rapid bony union ${ }^{7}$.

The objective of this study was to compare the operative outcome of patients with mild to moderate hallux valgus with a standard distal osteotomy vs SERI technique.

\section{Material And Methods}

We performed a prospective study of surgical cases from a single center. The local institutional review board approved this study. Between the years 2014-2015, one fellowship trained foot and ankle surgeon performed all procedures. Inclusion criteria for this study were: (1) patients with mild to moderate hallux valgus as defined by IMA $\geq 9^{\circ}$ and $<13^{\circ}$, (2) no previous surgical treatment for hallux valgus, (3) patients 18 years of age or greater, (4) patients indicated for procedures to address alterations in the IMA without simultaneous procedures addressing additional pathology.

One hundred and eight surgeries were performed for hallux valgus during the study period. Sixty-two patients were not eligible for randomization as they had additional pathology which required surgical intervention at the time of the procedure. Additionally, the first 10 patients who underwent a SERI technique correction of hallux valgus were excluded to account for the surgeon's learning curve. Ultimately, thirty-six patients who presented during the study period had mild-moderate hallux valgus without additional foot pathology requiring surgical correction and were eligible for inclusion (Flow Chart 1).

Patients indicated for isolated treatment of mild to moderate hallux valgus were randomized using a standard random number generator. The first patient randomized for the surgery day, SERI technique was performed. The surgeon then had time to template the procedure using the PACs system and templating software in the operating room prior to surgery. Twenty-one patients were selected for the SERI minimally invasive procedure and fifteen patients were selected for the standard Chevron osteotomy procedure.

\section{Preoperative Evaluation}


The diagnosis of mild to moderate hallux valgus was made via clinical evaluation and standing radiographs. The radiographic measurements and angles recorded included IMA, HVA, and DMMA (Figs. 1-2).

\section{Surgical Procedure}

\section{SERI Surgery Technique}

Surgery was performed under local anesthesia and an ankle block. Intravenous prophylactic antibiotics were given within 30 minutes prior to skin incision. The patient was positioned supine and the operative leg was prepared and draped in a sterile fashion. A $1.8 \mathrm{~mm}$ Kirshner Wire (KW) was introduced percutaneously to the medial and proximal nail fold, and manually advanced within the skin after crossing the prominent bunion. Subsequently, a proximal skin incision was performed, Periosteal stripping with a periosteal elevator around the neck of the first metatarsal was performed to preserve the soft tissue; Using a saw (STRYKER Corp.Kalamazoo, MI, USA) at a low velocity, a medial cortical metatarsal neck cut was made with the following angular modifications

The direction of the osteotomy was determined based on the previously used technique by Kadakia AR at $\mathrm{el}^{4}$. In order to avoid dorsal head elevation of the metatarsus, the osteotomy was oriented at $10-15^{\circ}$ in a plantar direction relative to a plane perpendicular to the metatarsal long axis, from dorsal-distal direction to plantar-proximal direction. And at $10-15^{\circ}$ from medial-proximal to lateral-distal direction, in order to prevent shortening of the metatarsal bone length during the recovery period (Fig. 3). Finally, the osteotomy was completed by a manual fracturing of the metatarsals' lateral cortex in order to preserve the vascular supply, followed by the insertion of a small curved grooved-lever osteotome into the medullary canal of the proximal metatarsal fragment (Fig. 4). Next, the metatarsal head was laterally displaced (Fig. 3) until a percutaneous medial KW could be advanced into the metatarsal medullary canal, adjacent to the grooved-lever osteotome (Fig. 4). The different steps of the procedure were done with the use of fluoroscopy to verify hardware positioning and desired fragments alignment. Finally, the small skin incision was irrigated and closed with a $2 / 0$ absorbable Vicry ${ }^{\text {TM } E T H I C O N ~ s u t u r e . ~ W o u n d ~}$ dressing was done using a gauze pad soaked in an iodine solution, over the metatarsus's lateral side to overcorrected position of the first toe. Similarly, a semilunar shaped gauze pad was added to the plantar area distally and proximally to the metatarsal cut, in order to decrease the risk of dorsal displacement of the fragment during the recovery period.

The chevron osteotomy was performed using a distal dorsomedial approach to the metatarsal head. When instability of the metatarsal head was observable, a 3.5 cannulated screw was inserted in order to secure the osteotomy.

\section{Post-operative Rehabilitation And Evaluation}


Patients in the SERI group and Chevron osteotomy group received a similar postoperative protocol to that described by Giannini et al ${ }^{1}$. After the operation, immediate weight bearing was allowed with a DARCO MedSurg $^{\text {TM }}$ shoe for 4 to 6 weeks, until signs of bone healing appeared on radiographs. Two weeks after the surgery, the wound was examined and redressed for an additional two weeks. A second postoperative visit at 4-6 weeks included wound examination and oblique and anterior-posterior (AP) standing radiographs of each foot to record the IMA, HVA, and DMMA (Figs. 1-2). Radiographs were additionally used to check for complications, such as osteonecrosis, non-union, or malunion. Three more consecutive postoperative visits were respectively performed at 3,6 , and 12 months, which also included standing radiographs of each foot. The KW was removed 4-5 weeks after surgery depending on the patient's age, smoking habits and radiographic results. An OrthoWedge ${ }^{\mathrm{TM}}$ Darco surgical shoe was worn for 6 weeks after the surgery and a regular shoe was permitted to be worn 1-2 weeks after the KW was removed. Patients completed the AOFAS scoring system at the final follow-up appointment.

\section{Statistical Analysis}

The statistical analysis was performed using a Fisher exact tests or a Chi-squared test at a significance level of P-value $<0.05$. All measured angles (IMA, HVA, and DMMA) were expressed as a mean and standard deviation of the mean. A one-way ANOVA to study the differences between preoperative and postoperative data was performed at follow-up. Statistical analysis was performed using IBM Statistical Package for the Social Sciences (SPSS) Statistics, version 21 for Windows (SPSS, Chicago, IL).

\section{Results}

Thirty-five patients underwent hallux valgus repair procedures between the years 2014-2015 and met all the inclusion criteria. The SERI group included 14 female and 6 male with a mean age of 38.7 (17-81) (Table 1A). The Chevron osteotomy included 15 patients (12 female and 3 male) with a mean age of 49.2 (15-85) (Table 1B). The mean age of the patients in the SERI group was 38.7 (Standard Error of the Mean [SEM] 5.15) and $49.2(\mathrm{SEM}=4.88)$ in the Chevron group, which was not significant different $(\mathrm{P}=$ 0.160). Demographic data are displayed in Table 1A and 1B.

At the one year follow up measurements of the IMA, HVA, and DMMA were performed. The IMA was measured post-operatively in each group and found to be $6.0^{\circ} \pm 2.3$ and $6.1^{\circ} \pm 3$ in the SERI group and Chevron group respectively $(P=0.930)$. The HVA was measured post-operatively in each group and found to be $20.1^{\circ} \pm 7.9$ and $20.7^{\circ} \pm 6.9$ in the SERI group and Chevron group respectively $(P=0.841)$. The DMMA was measured post-operatively in each group and found to be $6.4^{\circ} \pm 2.8$ and $7.5^{\circ} \pm 3.3$ in the SERI group and Chevron group respectively $(P=0.310)$. The metatarsophalangeal joint (MTPJ) motion was the only parameter found to be significantly different $(P<0.001)$ between groups at final follow up.

The exposure to fluoroscopy was measured using a mini C-arm system (Hologic Fluoroscan ${ }^{\circledR}$ InSight$\mathrm{FD}^{\mathrm{T}}$ ) in imaging snapshot mode during the different procedures. In the SERI group, a mean of 22.4 (12- 
52) intraoperative shots were used. In the Chevron group, a mean of $2.7(2-5)$ shots were used to evaluate the osteotomy.

In the SERI group, five patients (25\%) reported minor complications. Three patients (15\%) reported complications related to $\mathrm{KW}$ cite. One of which had the $\mathrm{KW}$ removed at 4 weeks for a decubitus injury and the second had a superficial pin site infection and was given ten days of oral antibiotics to treat a superficial wound infection. The third patient reported pain at the point of entry of the KW and no antibiotics or KW removal was indicated. One patient developed a dorsal bunion with an elevated 1st metatarsal (Fig. 5). The use of a silicon pad was required in a single foot due to mild toe crossover. Three patients (15\%) underwent additional procedures during follow up. A single foot (5\%) underwent new corrective surgery in another facility due to recurrence, discomfort, and dissatisfaction. One patient (5\%) underwent metatarsal phalangeal joint (MTPJ) fusion, which was performed by another surgeon at our institution. No signs of avascular necrosis (AVN) or osteoarthritis (OA) were found in the $x$-ray prior to the second surgery. Finally one patient underwent additional bunionectomy $(5 \%)$ two years after surgery. The SERI cohort did not report postoperative stiffness.

In the Chevron control group, four patients $(27 \%)$ required additional surgical procedures. One patient (6.7\%) underwent additional surgery for a prominent screw. One patient is scheduled for Akin osteotomy to address mild overlapping. Akin osteotomy was performed at a later stage in two patients (13.3\%) with mild remaining hallux valgus and were dissatisfied with their correction. No signs of non-union or AVN were detected at the one year follow up. After the Chevron osteotomy, patients complained of mild stiffness and was noted in the result of the AFOAS score in our patients. (Table 2) 
Table 1

A: SERI Group Patient Data and Measured Angles.

\begin{tabular}{|c|c|c|c|c|c|c|c|c|c|}
\hline $\begin{array}{l}\text { No } \\
\text { SERI } \\
\text { series }\end{array}$ & SEX & AGE & SIDE & $\begin{array}{l}\text { IMA } \\
\text { (before } \\
\text { surgery) }\end{array}$ & $\begin{array}{l}\text { HVA } \\
\text { (before } \\
\text { surgery) }\end{array}$ & $\begin{array}{l}\text { DMMA } \\
\text { (before } \\
\text { surgery) }\end{array}$ & $\begin{array}{l}\text { IMA } \\
\text { (after } \\
\text { surgery) }\end{array}$ & $\begin{array}{l}\text { HVA } \\
\text { (after } \\
\text { surgery) }\end{array}$ & $\begin{array}{l}\text { DMMA } \\
\text { (after } \\
\text { surgery) }\end{array}$ \\
\hline 1 & 2 & 33 & Right & 12.4 & 28.6 & 14.8 & 5.9 & 14.3 & 4.1 \\
\hline 2 & 2 & 53 & Left & 16.1 & 30.1 & 13.5 & 12 & 27.1 & 11.2 \\
\hline 3 & 2 & 17 & Left & 13.1 & 37 & 16.2 & 3.6 & 24 & 7.4 \\
\hline 4 & 2 & 17 & Right & 14.5 & 32.2 & 13.9 & 7.8 & 21.6 & 5.2 \\
\hline 5 & 2 & 18 & Left & 14.9 & 30.1 & 12.5 & 4.9 & 9.3 & 3.7 \\
\hline 6 & 2 & 76 & Right & 15.4 & 29.8 & 16.2 & 5.5 & 12.8 & 9.2 \\
\hline 7 & 1 & 17 & Right & 17 & 33.1 & 19.5 & 8.3 & 23.5 & 3.5 \\
\hline 9 & 1 & 20 & Left & 14.2 & 40 & 8.5 & 6.2 & 28 & 8.2 \\
\hline 10 & 1 & 72 & Left & 16.5 & 34 & 15.9 & 4.4 & 29.3 & 4.5 \\
\hline 11 & 2 & 19 & Right & 12.6 & 33.5 & 13.9 & 5.6 & 28.1 & 7.4 \\
\hline 12 & 2 & 54 & Left & 17.3 & 38.2 & 14.2 & 9.8 & 30 & 8.6 \\
\hline 14 & 1 & 21 & Right & 11.9 & 38 & 15.2 & 5.1 & 27 & 8.5 \\
\hline 15 & 2 & 64 & Right & 12.9 & 43 & 15.3 & 6.59 & 30 & 9.4 \\
\hline 16 & 2 & 56 & Left & 16.6 & 34.6 & 10.6 & 4.5 & 17.9 & 5.4 \\
\hline 17 & 2 & 37 & Right & 12.9 & 25 & 13.4 & 6.48 & 13.2 & 4.2 \\
\hline 18 & 2 & 17 & Left & 15 & 24.1 & 13.6 & 7.5 & 9.5 & 2.3 \\
\hline 19 & 1 & 23 & Right & 12.7 & 27.3 & 9.8 & 5.8 & 10.8 & 7.3 \\
\hline 20 & 2 & 59 & Right & 18.7 & 27.5 & 12.8 & 2.8 & 9.6 & 1.95 \\
\hline 22 & 2 & 81 & Left & 15.9 & 24.5 & 12.2 & 5.8 & 11.9 & 5.1 \\
\hline \multirow[t]{2}{*}{23} & 2 & 20 & Left & 14.4 & 29.2 & 15.4 & 2.4 & 24.8 & 10.8 \\
\hline & & $\begin{array}{l}\text { Mean: } \\
38.7 \\
\pm 23.0\end{array}$ & & $\begin{array}{l}\text { Mean: } \\
14.8 \pm \\
1.9\end{array}$ & $\begin{array}{l}\text { Mean: } \\
32.0 \pm \\
5.3\end{array}$ & $\begin{array}{l}\text { Mean: } \\
13.87 \pm \\
2.5\end{array}$ & $\begin{array}{l}\text { Mean: } \\
6.0 \pm \\
2.3\end{array}$ & $\begin{array}{l}\text { Mean: } \\
20.1 \pm \\
7.9\end{array}$ & $\begin{array}{l}\text { Mean: } \\
6 . \pm 2.8\end{array}$ \\
\hline
\end{tabular}


Table 2

Average of AOFAS Forefoot score

\begin{tabular}{|lllllllll|}
\hline Procedure & Pain & $\begin{array}{l}\text { Activity } \\
\text { limitation }\end{array}$ & $\begin{array}{l}\text { Foot } \\
\text { wear }\end{array}$ & $\begin{array}{l}\text { MTPJ } \\
\text { motion }\end{array}$ & $\begin{array}{l}\text { IPJ } \\
\text { motion }\end{array}$ & $\begin{array}{l}\text { Joint } \\
\text { stability }\end{array}$ & Callus & $\begin{array}{l}\text { Hallux } \\
\text { alignment }\end{array}$ \\
\hline SERI & 36.08 & 9.78 & 9.1 & 9.5 & 5 & 5 & 5 & 14 \\
\hline Chevron & 36.9 & 8.8 & 8.4 & 5.7 & 5 & 5 & 5 & 13.8 \\
\hline
\end{tabular}

Figure 1: Pre and Post-operative SERI patient

Figure 2: Pre and Post-operative Chevron Osteotomy

Figure 3: Dorsiflexed Metatarsal Head in SERI series

Figure 4: Modified osteototmy

Figure 5: Grooved-lever osteotome

Flow Chart 1:

\section{Discussion}

The purpose of surgical treatment of hallux valgus is to correct the deformity through a morphological and functional rebalance of the first ray and first MPJ, and to improve the patients' quality of life ${ }^{2}$. Various studies have demonstrated success with surgical treatment of mild to moderate hallux valgus regardless of surgical approach and the type of osteotomy or fixation technique. Their remains a paucity of sufficient data, clearly demonstrating the superiority of a single technique to address isolated mild to moderate hallux valgus pathology ${ }^{1-7}$.

The SERI technique was designed to avoid wound complications and reduce recovery time for patients with mild to moderate hallux valgus. In this study, 21 patients randomized to operative treatment with the SERI technique were assessed to evaluate the effectiveness of this treatment in terms of the techniques effectiveness, complication rate and the use of fluoroscopy. Both groups in this study demonstrated significant improvement in radiographic morphology postoperatively.

Kuhn et al ${ }^{8}$ studied a modified distal metatarsal osteotomy in 20 feet with a Chevron bunionectomy with adductor tenotomy with minimal stripping, and lateral release. Prior studies of this technique have demonstrated severe complications associated with this procedure, including osteonecrosis of the metatarsal head reported in up to $40 \%$ of patients ${ }^{1}$. In order to assess the blood flow, the Kuhn et al used doppler and demonstrated a $71 \%$ decrease in the blood flow to the operative area. Moreover, after the medial capsular release, the authors demonstrated a $45 \%$ decrease in blood flow to the metatarsal head. In the present study, there were no clinical or radiological finding of metatarsal head osteonecrosis. Care 
is taken during chevron osteotomy to spare the blood supply to the metatarsal head and the SERI technique is an MIS approach designed to minimize disruption of vascular supply.

Kadakia et al. ${ }^{4}$ evaluated a PDO in 13 feet and reported major complications of dorsal malunion in 9 feet $(69 \%)$ and recurrent hallux valgus in 5 feet (38\%). Mittag et al. ${ }^{9}$ assessed a proximal metatarsal osteotomy with a distal soft-tissue procedure in 151 feet and reported a non-union in 4 feet (2.6\%). These complications may be secondary to insufficient internal fixation $4 ; 9$. In our study, there were no occurrences of non-union. The SERI's plantar inclination is designed for inherent construct stability and improved weight distribution of the metatarsal head, which encourages healing. The technique relies on a single KW for fixation and despite this our cohort did not experience osteotomy displacement. However, close patient follow-up is essential to avoid recurrence and complications associated with a percutaneous KW fixation. In this study, the AOFAS score validated that the SERI technique can be effective in relieving the symptoms and improving the function of the first toe. Additional imaging showed the efficacy of the SERI technique in treating the morphologic deformities in minor and moderate hallux valgus (IMA, HVA, DMAA).

Park et al. ${ }^{10}$ assessed the effectiveness of a proximal Chevron osteotomy with a distal soft-tissue technique for treatment of hallux valgus in 117 feet. Twenty feet $(17.1 \%)$ reported recurrence with follow 24 months of follow up. These recurrences were believed to be secondary to sesamoid malposition. In the SERI group of the present study, a recurrence rate of $4.3 \%$ was recorded.

To ensure effectiveness of the SERI technique, close post-operative follow-up is necessary in order to ensure the reduction has not been lost, monitor for pin site infection or decubitus ulcers about the pin site. The SERI technique is relatively simple because it involves only a single KW for fixation and does not require lateral release or removal of the medial eminence. Nonetheless, the technique proved to be effective and provide good results in this randomized controlled trial with minimum one year follow up. Operative time for the SERI procedure is approximately 7-15 minutes, with an additional 5-7 minutes for closure and to apply the bandage. The SERI technique is inexpensive as surgical time is rapid, it only uses one KW for fixation, and it does not require specialized instruments. The most significant advantages for this technique are reduced surgical time, minimized soft tissue injury, and high rates of patient satisfaction $1 ; 2 ; 5 ; 7 ; 9-12$. These advantageous features make the SERI technique an effective tool for medically compromised patients, such as those with diabetes mellitus and hallux valgus deformities.

\section{Limitations}

This study presented with several limitations. Data were recorded from a small patient population and the SERI technique is only suitable for minor and moderate hallux valgus pathology. Pre-operatively, the AOFAS score was not recorded limiting the ability to assess improvement in AOFAS scores. The learning curve associated with performing a new procedure constitutes another limitation. However, in this study the first ten cases the surgeon performed using this technique were excluded. While HVA and IMA can be accurately recorded using a radiograph, radiographic assessment poses a limitation as it is difficult to 
diagnose the DMAA in this manner as it has poorer observer reliability ${ }^{13 ; 14}$. While this study was conducted as a randomized control trial (RCT), the cohort was not large enough to be a true RCT and additional larger RCTs should be conducted in the future.

\section{Conclusion}

This prospective trial demonstrated that the SERI technique for the treatment of mild and moderate hallux valgus may be an effective, safe, and easily reproducible surgery that provides results comparable to a chevron osteotomy without and increased complication rate. Surgeons who use this technique should be aware of specific concerns associated with a performing a plantar angled osteotomy, and should consider close postoperative monitoring.

\section{Abbreviations}

*AOFAS - American Orthopedic Foot and Ankle Society

*AP - Anterior-posterior

*AVN - Avascular Necrosis

*DMAA - Distal Metatarsal Articular Angle

*HVA - Hallux Valgus Angle

*IMA - Intermetatarsal Angle

*KW - Kirshner Wire

*MTPJ - Metatarsophalangeal Joint

*OA - Osteoarthritis

*PDO - Percutaneous distal metatarsal osteotomy

*SERI -Simple, Effective, Rapid, Inexpensive

*SSPS - Statistical Package for the Social Sciences

\section{Declarations}

Ethics approval and consent to participate: The study was approved by the local institutional review board.

Consent for publication: Not applicable. 
Availability of data and material: Not applicable.

Competing interests: The authors declare that they have no competing interests.

Funding: Not applicable.

Authors' contributions: EP and ON collected the data; EP, RA analyzed and interpreted the x-ray images; EP, AR, ST and HI were the major contributors in writing the manuscript; NO and AE reviewed the manuscript. All authors read and approved the final manuscript.

Acknowledgements: We thank Mr. Nitzan Konstantin for assistance with writing the manuscript

\section{References}

1. Giannini S, Faldini C, Nanni M, et al. 2014. Reply to comments by Wu et al. regarding our manuscript titled: A minimally invasive technique for surgical treatment of hallux valgus: simple, effective, rapid, inexpensive (SERI). Int Orthop 38:673-674.

2. Maffulli N, Longo UG, Marinozzi A, et al. 2011. Hallux valgus: effectiveness and safety of minimally invasive surgery. A systematic review. Br Med Bull 97:149-167.

3. Bösch P, Wanke S, Legenstein R. 2000. Hallux valgus correction by the method of Bösch: a new technique with a seven-to-ten-year follow-up. Foot and ankle clinics 5:485-498, v.

4. Kadakia AR, Smerek JP, Myerson MS. 2007. Radiographic results after percutaneous distal metatarsal osteotomy for correction of hallux valgus deformity. Foot Ankle Int 28:355-360.

5. Nyska M. 2001. Principles of first metatarsal osteotomies. Foot Ankle Clin 6:399-408.

6. Palmanovich E, Myerson MS. 2014. Correction of moderate and severe hallux valgus deformity with a distal metatarsal osteotomy using an intramedullary plate. Foot Ankle Clin 19:191-201.

7. Trnka HJ, Krenn S, Schuh R. 2013. Minimally invasive hallux valgus surgery: a critical review of the evidence. Int Orthop 37:1731-1735.

8. Kuhn MA, Lippert FG, 3rd, Phipps MJ, et al. 2005. Blood flow to the metatarsal head after chevron bunionectomy. Foot Ankle Int 26:526-529.

9. Mittag F, Leichtle U, Meisner C, et al. 2013. Proximal metatarsal osteotomy for hallux valgus: an audit of radiologic outcome after single screw fixation and full postoperative weightbearing. $J$ Foot Ankle Res 6:22.

10. Park CH, Lee WC. 2017. Recurrence of Hallux Valgus Can Be Predicted from Immediate Postoperative Non-Weight-Bearing Radiographs. J Bone Joint Surg Am 99:1190-1197.

11. Maffulli N, Oliva F, Coppola C, et al. 2005. Minimally invasive hallux valgus correction: a technical note and a feasibility study. J Surg Orthop Adv 14:193-198.

12. Roukis TS. 2009. Percutaneous and minimum incision metatarsal osteotomies: a systematic review. The Journal of foot and ankle surgery : official publication of the American College of Foot and Ankle Surgeons 48:380-387. 
13. Chi TD, Davitt J, Younger A, et al. 2002. Intra- and inter-observer reliability of the distal metatarsal articular angle in adult hallux valgus. Foot Ankle Int 23:722-726.

14. Easley ME, Trnka HJ. 2007. Current concepts review: hallux valgus part 1: pathomechanics, clinical assessment, and nonoperative management. Foot Ankle Int 28:654-659.

\section{Figures}

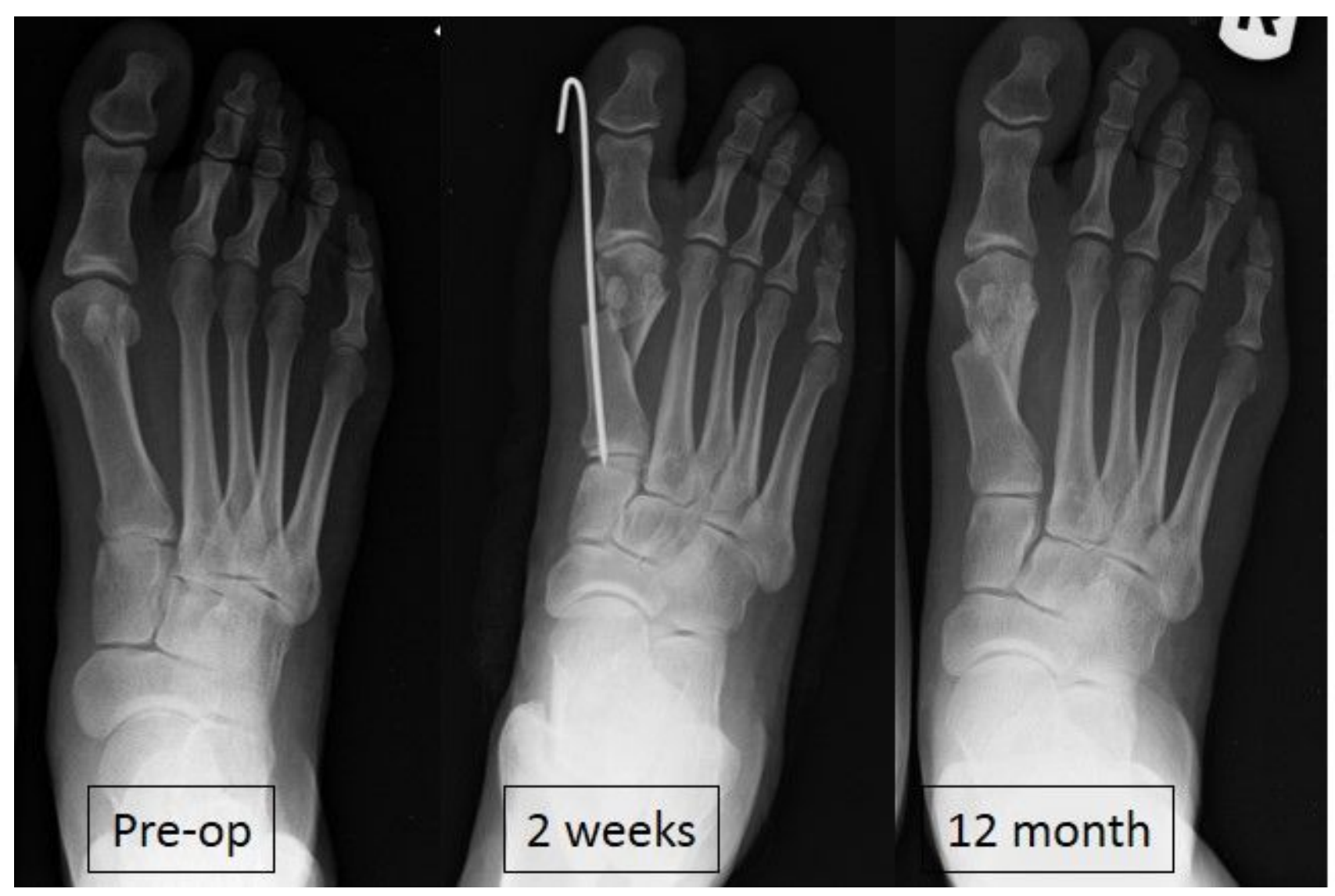

\section{Figure 1}

Pre and Post-operative SERI patient 

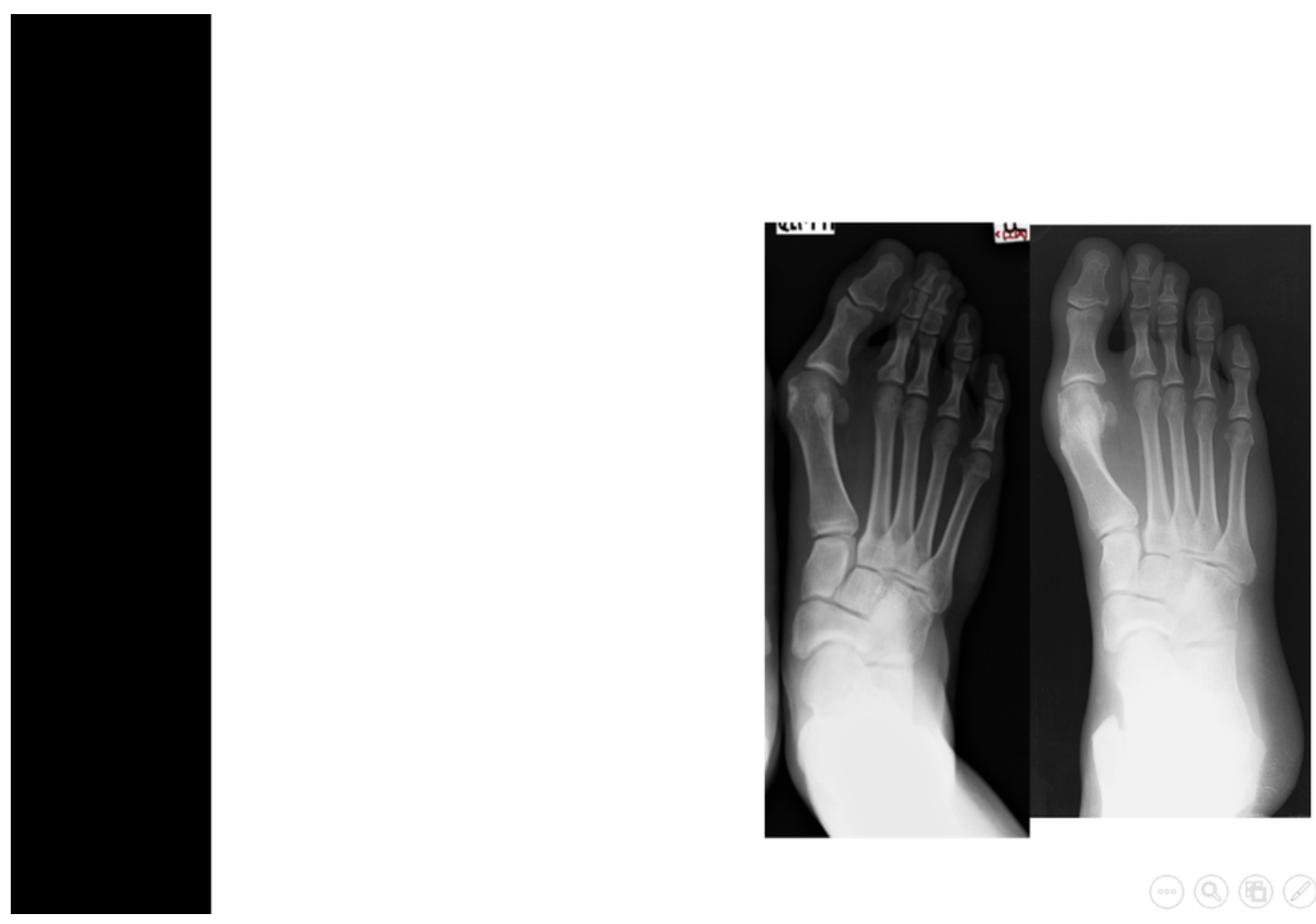

Figure 2

Pre and Post-operative Chevron Osteotomy 


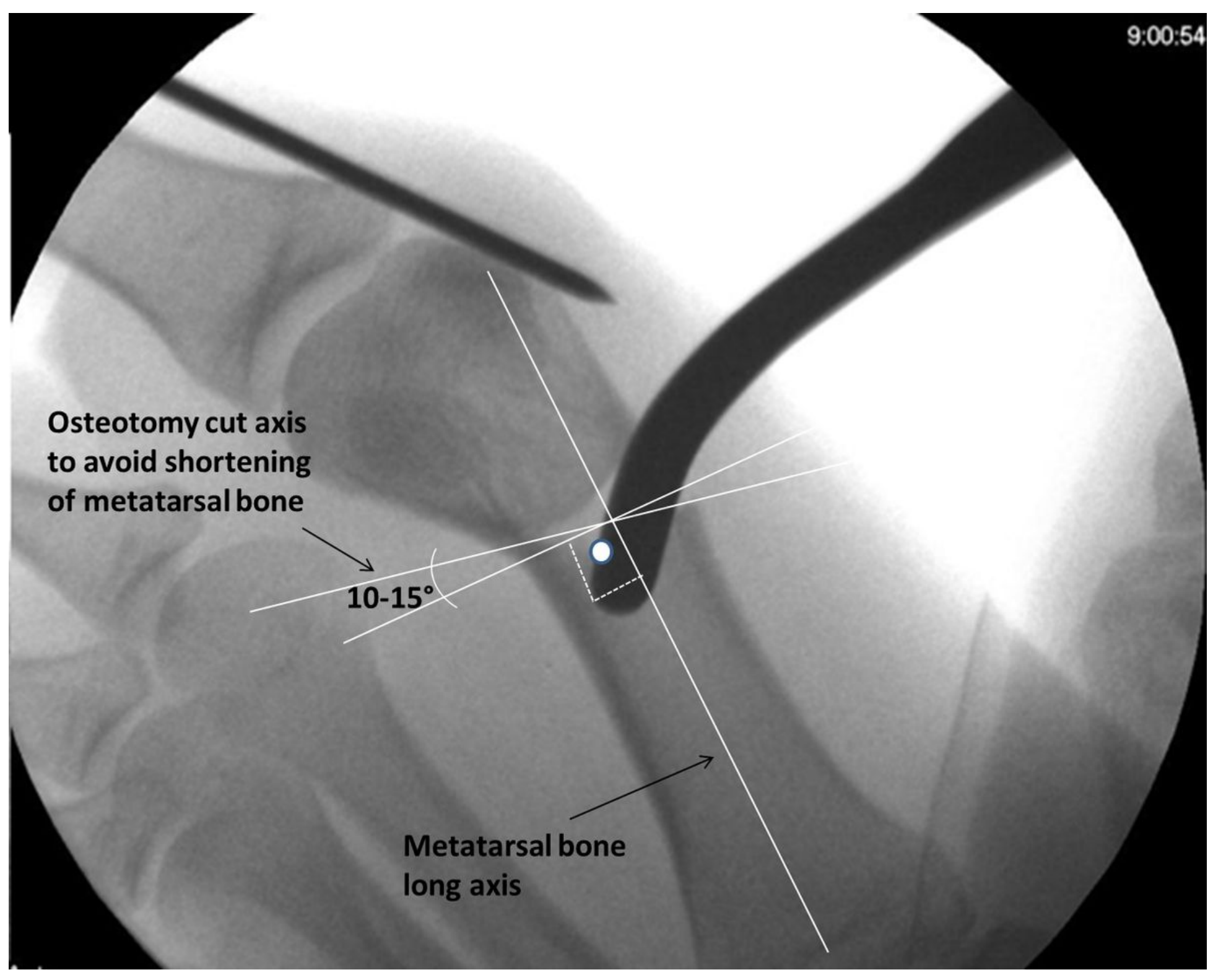

Figure 3

Dorsiflexed Metatarsal Head in SERI series 


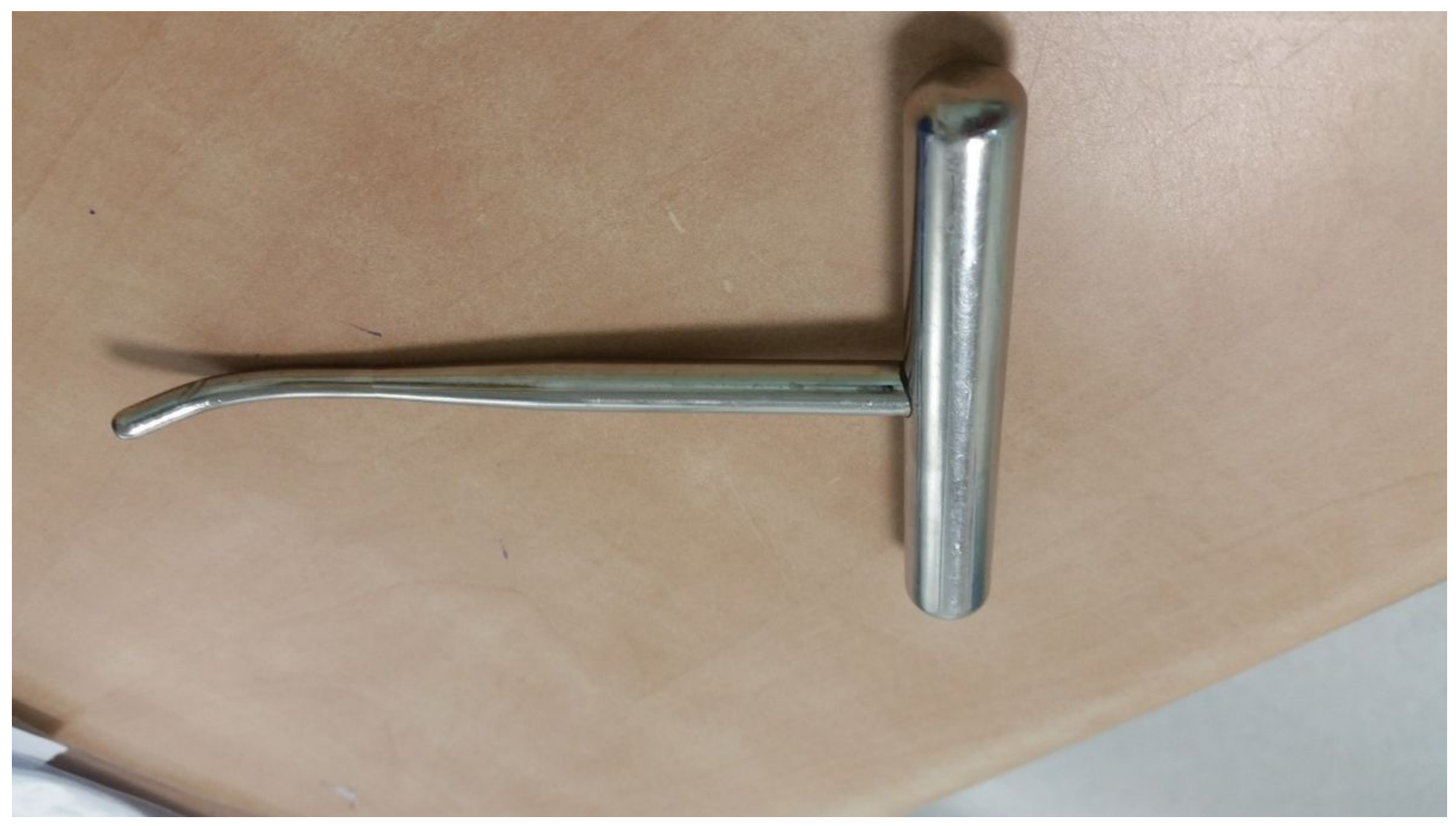

Figure 4

Modified osteototmy
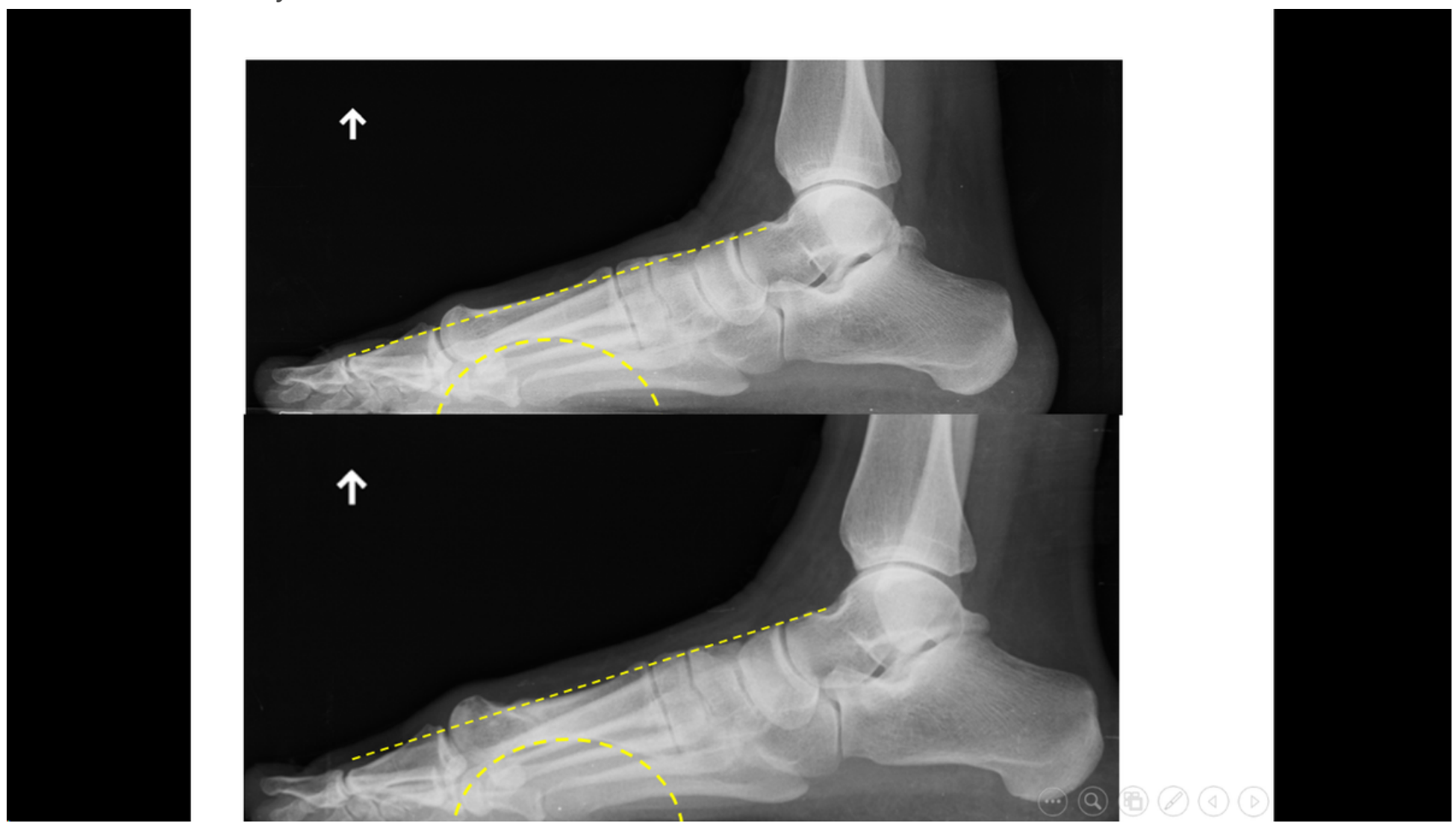
Figure 5

Grooved-lever osteotome

\section{Supplementary Files}

This is a list of supplementary files associated with this preprint. Click to download.

- FlowChart.jpg 